\title{
Magnitude of Undernutrition and associated factors among children with cardiac disease in University of Gondar hospital, northwest Ethiopia
}

\author{
Mulat Asrade \\ University Of Gondar \\ Abdulkadir Shehibo ( $\sim$ abduye2002@gmail.com ) \\ University Of Gondar \\ Zemene Tigabu \\ University Of Gondar
}

\section{Research Article}

Keywords: Undernutrition, malnutrition, cardiac, Gondar, Ethiopia.

Posted Date: February 26th, 2021

DOI: https://doi.org/10.21203/rs.3.rs-194650/v1

License: (1) This work is licensed under a Creative Commons Attribution 4.0 International License.

Read Full License

Version of Record: A version of this preprint was published at BMC Nutrition on August 5th, 2021. See the published version at https://doi.org/10.1186/s40795-021-00449-9. 


\section{Abstract}

Background: Undernutrition and cardiac disease are interconnected in a vicious cycle. Children with cardiac disease suffer with different degree of undernutrition resulting in significant morbidity and mortality in developing countries. This study aimed to assess magnitude of undernutrition and associated factors among children with cardiac disease in University of Gondar hospital, northwest Ethiopia.

Method: Hospital based quantitative cross-sectional study conducted among 269 children with cardiac disease visiting University of Gondar specialized hospital, northwest Ethiopia. Data was collected by using a pretested self-administered structured questionnaire and medical records of the patients. All participants who fulfill the inclusion criteria were included. The data analysed by using binary logistic regression model. Variables with $\mathrm{p}$-value $\leq 0.2$ in bivariate analysis were fitted to the final multivariable analysis and those variables with $p$-value $\leq 0.05$ were considered as having statistically significant association to the outcome variable. AOR and $95 \%$ confidence interval was calculated to assess the strength of association between the variables.

Result: Of the 269 study participants, $177(65.7 \%)$ of them were having undernutrition. $54.5 \%$ were underweight, $39.7 \%$ were stunted, and $53.9 \%$ were wasted. The result showed that having pulmonary hypertension[AOR $=3.82,(95 \% \mathrm{Cl} 1.80,8.10)]$, NYHA/modified Ross class III and IV heart failure[AOR=4.64 $(1.69,12.72)]$ and cardiac chamber enlargement $[\mathrm{AOR}=2.91(1.45,5.66)]$ were significantly associated with undernutrition.

Conclusion: There is high burden of undernutrition among children with cardiac diseases in the study area. Nutritional management and dietary counseling besides early detection and treatment of the underlying heart disease improves the nutritional status of children with cardiac diseases.

\section{Introduction}

Undernutrition remains one of the most common causes of morbidity and mortality among children throughout the world. Globally, moderate acute undernutrition affects 32.8 million children and severe acute undernutrition affects for 18.7 million children(1). Assuming every cases of undernutrition as lack of availability of food due to poor socio-economic status is wrong and may be associated with adverse outcomes(2). Undernutrition could be secondary to several underlying medical disease or conditions or these diseases may be a contributing factor. Cardiovascular diseases are one of the commonest medical conditions which are strongly associated with varying degrees of undernutrition(3-5).

The relationship between underlying cardiac disease and undernutrition is multidirectional. Undernutrition is strongly associated with frequent hospitalization, recurrent infections, poor postoperative outcome and increased mortality. However, the cause of undernutrition in cardiac patients is multifactorial. Cardiac diseases can cause or worsen undernutrition due to several reasons. These could be due to decreased 
food intake, increased energy requirement and venous congestion of the bowel resulting in poor nutrient absorption (6-9).

Children with genetic syndromes/chromosomal disorders, advanced heart failure and pulmonary hypertension are more prone to develop growth failure and undernutrition (9-12). Every child with cardiac diseases should be screened for growth failure and undernutrition to identify patients at high risk of poor outcomes who might benefit either from medical management or surgical interventions to prevent deterioration of congestive heart failure and improve prognosis(13). Nutritional status assessment of cardiac patients is often neglected in majority hospitals in Ethiopia, despite the undernutrition contributed for increased morbidity and mortality of our cardiac patients. Therefore, the study aimed to determine the burden and associated factors undernutrition among children with cardiac disease in Gondar comprehensive specialized hospital, northern Ethiopia. The findings of this study can help for health managers and policy makers to set recommendations and public health intervention for nutritional screening and nutritional supplementation among children with cardiac illness.

\section{Methods}

An institution based cross-sectional study was conducted in the pediatric cardiac clinics of university of Gondar comprehensive and specialized hospital. University of Gondar Hospital is a resource-limited a teaching and tertiary care hospital in northern Ethiopia. It receives referrals from an area that encompasses more than 17 million people.

Data was collected between April 1, to June 30, 2019. A sample size of 273 was calculated assuming a $50 \%$ prevalence rate of undernutrition (given no previous baseline population), a $5 \%$ margin of error, source population $<10,000$ and a $10 \%$ non-response rate at $95 \%$ confidence interval. Patients were included in the study had echocardiographic evidence of anatomic and/or functional heart defect that existed before or after birth and they were not older than 18 years of age. Children with prematurity, a known genetic or chromosomal disorder, and chronic illness other than cardiac disease associated with growth failure and undernutrition were excluded.

Socio-demographic, anthropometric measurement, clinical and echocardiographic data were collected using a structured, pretested questionnaire. Data collectors were a trained pediatric residents and general practitioner who were working in the pediatric cardiac clinic. In every case cardiac diagnosis was made based on clinical evaluation and investigation modalities like Doppler echocardiography. Pulmonary hypertension was defined using transthoracic echocardiography.

The nutritional status of the study participants was assessed using the standard indicators of weight-forage (WFA), height-for-age (HFA), weight-for-height (WFH) and mid upper arm circumference (MUAC) and anthropometric measurement interpreted using World health organization (WHO) reference standards to categorize nutritional status. The WHO global database on undernutrition recommends a cut-off $\mathrm{z}$ score of $\leq-2$ to classify low WHZ (wasting), low WAZ (underweight) and low HAZ (stunting) as moderate undernutrition, and a $z$ score of $\leq-3$ SD to define severe undernutrition. 
Data obtained from the study was entered, cleaned \& verified using Epi-info 7. Then, the data were exported to SPSS version 22.0 for analysis. Descriptive summary like frequencies, proportions, graphs and cross tabs were used to present the study result. Bivariate and multivariate logistic regression analyses were conducted to determine the effect of factor(s) on the outcome variable representing undernutrition and to control possible confounders. Statistical association was checked by $95 \%$ confidence interval and crude odd ratio. Later, the significant variables ( $p$-value $<0.2$ ) observed in bivariate analysis were subsequently included in multivariate analysis. Finally, 95\% confidence interval and adjusted odd ratio were checked and the significance variables were taken as associated of undernutrition. All methods were performed in accordance to declaration of Helenski. Informed consent was obtained from parents/legal guardians and Ethics approval for this study was obtained from internal review boards at GUH in Gondar, Ethiopia

\section{Results}

A total of 269 children with cardiac disease were included in this study. $142(52.8 \%)$ patients were female with a male to female ratio nearly 1:1. The mean age was 9.64 year with a range of 1 month to 18 years. Half of the patients were under five children. Majority were from parent who did not attend school. These data are presented in table 1.

Table 1 Socio-demographic characteristics of children with cardiac disease $(\mathrm{No}=269)$ 


\begin{tabular}{lll}
\hline Variable & Frequency & Percent (\%) \\
\hline Sex & 142 & 52.8 \\
Female & 127 & 47.2 \\
Male & & \\
Residence & 140 & 52 \\
Rural & 129 & 48 \\
Urban & & \\
Age (in months) & 62 & 23 \\
<24 & 72 & 26.8 \\
24-59 & 87 & 32.3 \\
60-143 & 48 & 17.8 \\
>=144 & & \\
Maternal occupation & 30 & 11.2 \\
Employed & 178 & 66.2 \\
Housewife & 21 & 7.8 \\
Merchant & 40 & 14.8 \\
Daily laborer\& others & & \\
Maternal education & 143 & 53.2 \\
Unable to read\& write & 42 & 15.6 \\
able to read\& write & 37 & 13.8 \\
Primary education & 29 & 10.7 \\
Secondary education & 18 & 6.7 \\
Tertiary education \&above & & \\
Father education & & 37.5 \\
Uneducated & 101 & 22.7 \\
Able to read and write & 61 & 17.8 \\
Primary education & 48 & 8.2 \\
Secondary education & 22 & 13.8 \\
Tertiary education \&above & 37 & 17.1 \\
Number of family members & & 41.2 \\
<=3 & 46 & 24.2 \\
4-5 & 111 & 17.5 \\
6-7 & 65 & \\
8 and above & 47 & \\
\hline
\end{tabular}

Acquired heart disease was much common than congenital heart disease (69.5\% Vs $30.1 \%$ ), and $16.4 \%$ of them had echocardiographic report of pulmonary hypertension. Rheumatic heart disease was the most common form of acquired heart disease in the pediatric follow up clinic during the study period.136 had congestive heart failure heart failure and $90.3 \%$ of children with those cardiac disease had history of hospitalization due to several reasons. Of the total congenital heart disease, $68.2 \%$ had Acyanotic congenital heart disease and $31.8 \%$ had cyanotic congenital heart disease (table 2 ).

Table 2: Clinical profile of children with cardiac disease at University of Gondar specialized referral hospital, northwest Ethiopia, $2019(\mathrm{No}=269)$ 


\begin{tabular}{lll}
\hline Variables & Frequency & Percent (\%) \\
\hline Duration of symptom before Diagnosis (in months) & & \\
$<6$ & 192 & 71.4 \\
$>=6$ & 77 & 28.6 \\
Co morbid disease & & \\
$\quad$ Yes & 39 & 14.5 \\
$\quad$ No & 230 & 85.5 \\
Frequency of follow up & & \\
$\quad$ Monthly & 166 & 61.7 \\
Every 2month & 88 & 32.7 \\
$\quad$ Every 3month and above & 15 & 5.6 \\
Heart failure & & \\
No & 133 & 49.4 \\
$\quad$ Mild & 78 & 29 \\
Moderate to severe & 58 & 21.6 \\
Chamber enlargement & & \\
Yes & 159 & 59.1 \\
$\quad$ No & 110 & 40.9 \\
Pulmonary hypertension & & \\
Yes & 44 & 16.4 \\
No & 225 & 83.6 \\
Type of cardiac disease & & \\
$\quad$ Congenital & 82 & 30.5 \\
Acquired & 187 & 69.5 \\
$\quad$ & & \\
\hline
\end{tabular}

The prevalence of undernutrition was found to be $65.7 \%$, of this $34.5 \%$ had moderate acute undernutrition and $31.2 \%$ severe acute undernutrition. 107 (39.7\%) of patients had stunting, of this $25.2 \%$ were moderately stunted and $14.5 \%$ severely stunted. The prevalence of underweight and wasting was $54.5 \%$ and $53.9 \%$ respectively. The prevalence of undernutrition was higher in children with congenital heart disease as compared to acquired heart disease (figure 1).

In the multivariable analysis, pulmonary hypertension, moderate to severe heart failure and echocardiographic evidence of cardiac chamber enlargement were significantly associated with undernutrition. Children with moderate to severe heart failure were $4.64(95 \% \mathrm{Cl}: 1.69,12.72)$ times more malnourished as compared with children with no heart failure. The result also indicated that the magnitude of undernutrition among children with pulmonary hypertension [AOR=3.79; 95\% Cl: 1.10, 13.1], and echocardiographic evidence of cardiac chamber enlargement [AOR=2.91; $95 \mathrm{Cl}: 1.45,5.66]$ were significantly associated in the study (table 3 ).

Table-3. Association of undernutrition among children with cardiac diseases at University of Gondar referral hospital, northwest Ethiopia, 2019(No=269). 


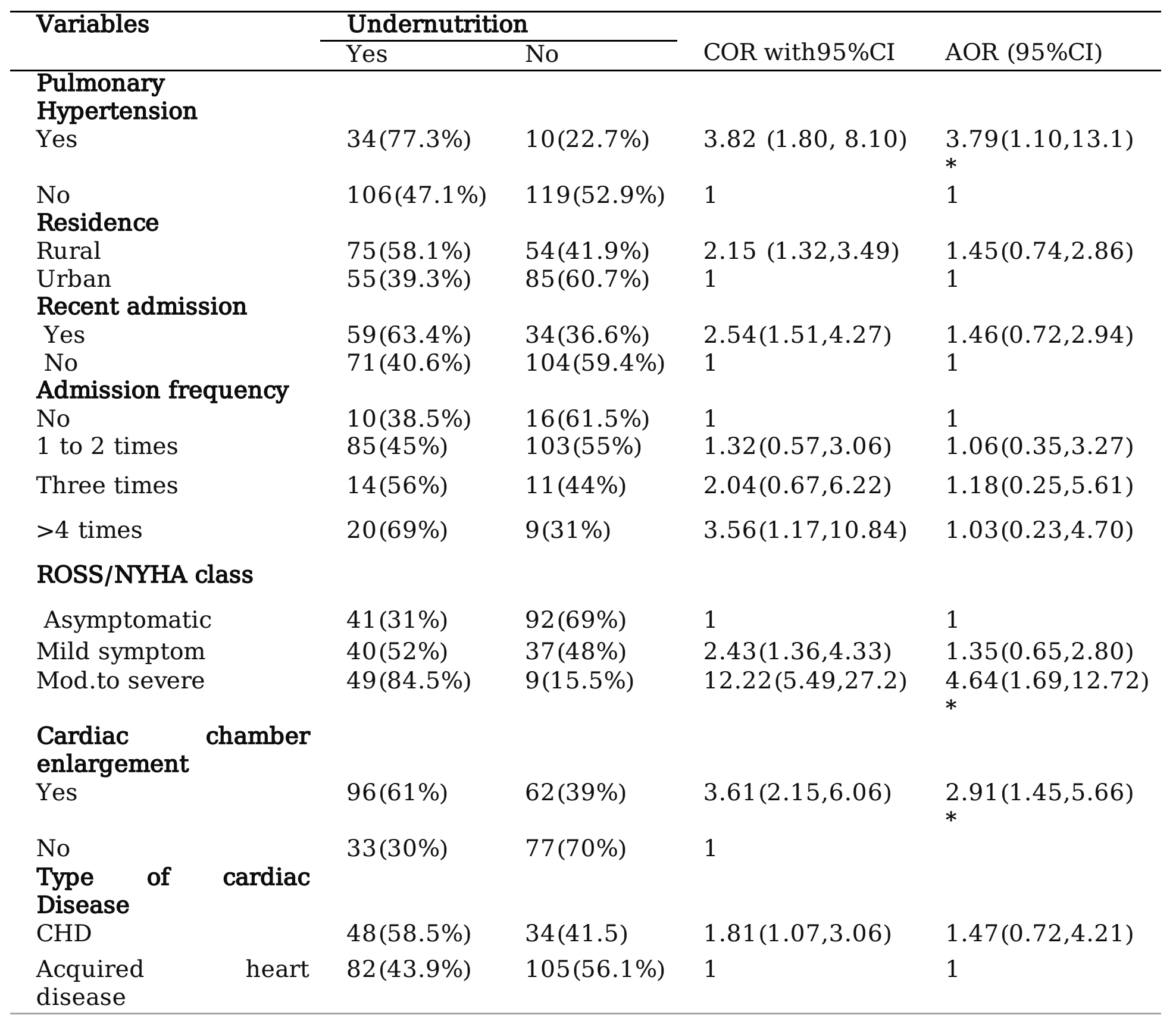

\section{Discussion}

It is well known that undernutrition is common in cardiac patients and related with increased morbidity and mortality. In developing countries like Ethiopia where surgical intervention for cardiac disease like congenital heart disease and/or rheumatic heart disease is scarce or unavailable at all, the magnitude of undernutrition is expected to be high.

This study reports a high burden of undernutrition and growth failure in children with cardiac diseases. The overall prevalence of undernutrition was $65.7 \%$, with $31.2 \%$ of cases having severe acute undernutrition. Stunting which is an indicator of chronic undernutrition was found to be $39.7 \%$ and with $14.5 \%$ of cases had severe stunting. As noted above, this study reported a very high prevalence of 
undernutrition in children with cardiac disease as compared to the country national estimate of undernutrition in Ethiopia(14). Other studies also showed children with cardiac disease are higher risk of undernutrition compared to those without cardiac disease(10).

Similar to our finding, a study done in turkey by Varun etal reported higher prevalence of acute undernutrition (65\%) and chronic undernutrition (42\%)(7). Okoromah and colleagues reported a prevalence of undernutrition (90.4\%), severe undernutrition (61.2\%) and chronic undernutrition indicated by stunting $(28.8 \%)$ in children with congenital heart disease visiting a tertiary teaching hospital Lagos, Nigeria. Though they reported lower prevalence of stunting, the overall prevalence of undernutrition and severe acute undernutrition is much higher than those seen in our study(6). Another study in India done by Vaidyanathan and colleagues, shows higher prevalence of acute undernutrition (55.9\%) evidenced by weight for height(15). This suggests the presence of heterogeneity from country-to-country accounting for the difference in determinant of undernutrition among children with cardiac disease.

Based on the multivariable analysis we have found that the likelihoods of undernutrition were significantly higher among children with NYHA/Modified ROSS class III and IV heart failure, cardiac chamber enlargement and pulmonary hypertension. This is in line with various studies that reported children with advanced congestive heart failure and/or pulmonary hypertension were more likely to be malnourished $(9,10,16)$. An explanation might be the fact that children with heart failure and pulmonary hypertension could have poor oral intake and also congestion of bowel and liver attributing to early satiety. In addition to this, heart failure causes a cascade of sympathetic activation and leading the patient to a hypermetabolic state with higher caloric demand(17).

This study has certain limitations. First, this a hospital-based study and there is high probability of patients are referred to us with advanced heart failure resulting in high burden of undernutrition Thus, our patients are not likely to be representative of the full extent of the disease prevalence at the community level. Second, the small proportion of children with cardiac disease in our study may bias the associations, in that we may have failed to detect significant associations based on proportions, which may have existed. However, this study provides new insights into the burden of undernutrition and its associated factors among children with cardiac disease in our hospital and encourages for a more comprehensive population-based analysis.

Conclusion and Recommendation: Undernutrition is a very common morbidity among children with cardiac disease. Advanced congestive heart failure, presence of pulmonary hypertension and cardiac chamber enlargement were associated with undernutrition. We recommend routine nutritional counseling and nutritional rehabilitation as standard practice for all children with cardiac disease. In addition to this we recommend every effort to be made for early and definitive corrective measures to be performed including surgery.

\section{Declarations}


We acknowledge Mr Geta Asrade for their critical comments and technical contribution during design of the study. We would also like to thank University of Gondar for providing ethical clearance.

\section{Authors' contributions}

MA had designed the study, collected the data, and involved in data analysis and interpretation. AS and ZT had assisted in the data analysis, model development and critically commented and approved the final paper. All authors read and approved the final version of the manuscript.

\section{Consent to publication}

Not applicable

\section{Ethical approval and consent to participate}

Ethics approval for this study was obtained from internal review boards at GUH in Gondar, Ethiopia and Informed consent was obtained parents/legal guardians.

\section{Availability of Data and Materials}

All relevant data are available within the manuscript

\section{Competing interests}

The authors declare that they have no competing interests.

\section{Authors information}

MA is an assistant professor of pediatrics and child health, collage of medicine and health science at university of Gondar, Ethiopia. AS is an assistant professor of pediatrics and child health collage of medicine and health science at university of Gondar, Ethiopia. AS is also a consultant pediatric cardiologist, at university of Gondar. ZT is an associate professor of pediatrics and child health collage of medicine and health science at university of Gondar, Ethiopia. ZT is also a senior consultant pediatric cardiologist, at university of Gondar.

\section{Funding}

No funding

\section{References}

1. Lenters L, Wazny K, Bhutta ZA. Management of Severe and Moderate Acute Undernutrition in Children. Edition (Volume 2).

2. Shrivastava S. Undernutrition in congenital heart disease: Indian Pediatr. 2008 Jul;45(7):535-6. 
3. Amare $\mathrm{H}$, Hamza L, Asefa $\mathrm{H}$. Undernutrition and associated factors among heart failure patients on follow up at Jimma university specialized hospital, Ethiopia. BMC Cardiovasc Disord. 1186;15(128):015-0111.

4. Jain D, Rao SK, Kumar D, Kumar A, Sihag BK. Cardiac changes in children hospitalized with severe acute undernutrition: A prospective study at tertiary care center of northern India. Indian Heart J. 1016;71(6):492-5.

5. Mahdavi-Roshan M, Salari A, Hasandokht T, Rahbar-Taramsari M. Undernutrition Status and Associated Factors in Cardiac Patients Admitted to Heart Hospital, in the North of Iran. Hosp Top. 1080;97(2):60-5.

6. Okoromah CA, Ekure EN, Lesi FE, Okunowo WO, Tijani BO, Okeiyi JC. Prevalence, profile and predictors of undernutrition in children with congenital heart defects: a case-control observational study. Arch Dis Child. 1136;96(4):354-60.

7. Varan B, Tokel K, Yilmaz G. Undernutrition and growth failure in cyanotic and acyanotic congenital heart disease with and without pulmonary hypertension. Arch Dis Child. 1999;81(1):49-52.

8. Batte A, Lwabi P, Lubega S, Kiguli S, Otwombe K, Chimoyi L, et al. Wasting, underweight and stunting among children with congenital heart disease presenting at Mulago hospital, Uganda. BMC Pediatr. 1186;17(1):017-0779.

9. Arodiwe I, Chinawa J, Ujunwa F, Adiele D, Ukoha M, Obidike E. Nutritional status of congenital heart disease (CHD) patients: Burden and determinant of undernutrition at university of Nigeria teaching hospital Ituku - Ozalla, Enugu. Pak J Med Sci. 1140;31(5):1140-5.

10. Zhang M, Wang L, Huang R, Sun C, Bao N, Xu Z. Risk factors of undernutrition in Chinese children with congenital heart defect. BMC Pediatr. 1186;20(1):020-02124.

11. Blasquez A, Clouzeau H, Fayon M, Mouton JB, Thambo JB, Enaud R, et al. Evaluation of nutritional status and support in children with congenital heart disease. Eur J Clin Nutr. 1038;70(4):528-31.

12. Tevik K, ThÃ $1 / 4$ rmer $H$, Husby MI, de Soysa AK, Helvik AS. Nutritional risk is associated with long term mortality in hospitalized patients with chronic heart failure. Clin Nutr ESPEN. 1016;2016(12):e20-e9.

13. Sze S, Pellicori P, Kazmi S, Rigby A, Cleland JGF, Wong K, et al. Prevalence and Prognostic Significance of Undernutrition Using 3 Scoring Systems Among Outpatients With Heart Failure: A Comparison With Body Mass Index. JACC Heart Fail. 1016;6(6):476-86.

14. Zerfu T. Ethiopian Demographic and Health Survey, 2016.

15. Vaidyanathan B, Nair SB, Sundaram KR, Babu UK, Shivaprakasha K, Rao SG, et al. Undernutrition in children with congenital heart disease (CHD) determinants and short term impact of corrective intervention. Indian Pediatr. 2008;45(7):541-6.

16. Staebel 0 . Undernutrition in Belgian children with congenital heart disease on admission to hospital. Journal of Clinical Nursing. 2001;9:784-91.

17. Agra Bermejo RM, GonzÃ ilez Ferreiro R, Varela RomÃ $i n$ A, GÃ ${ }^{3} m e z$ Otero I, Kreidieh O, Conde SabarÃs $P$, et al. Nutritional status is related to heart failure severity and hospital readmissions in acute heart failure. Int J Cardiol. 1016;230:108-14. 
Figures

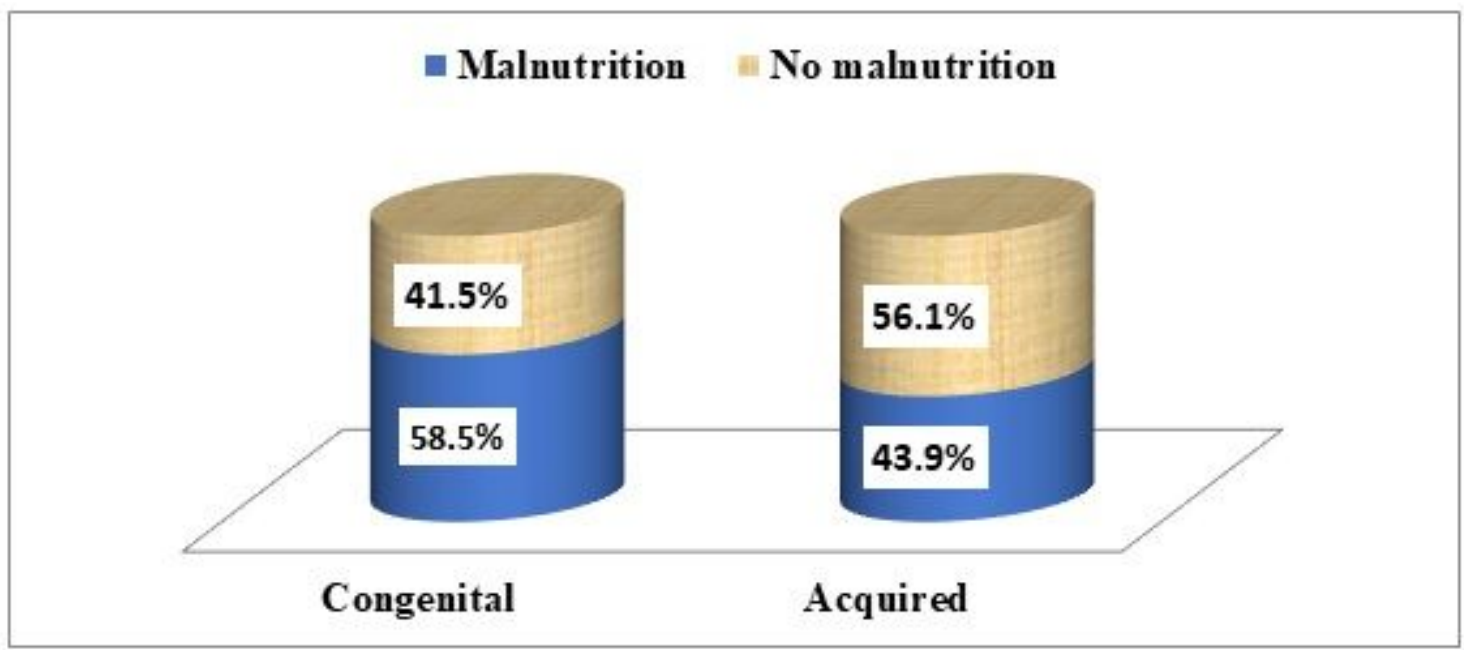

\section{Figure 1}

Nutritional status of children with cardiac disease (congenital versus acquired) at University of Gondar specialized referral hospital, northwest Ethiopia, 2019 (No=269) 